\title{
Combinación de entrenamiento de fuerza y aeróbico en adultos mayores: efectos en rendimiento funcional, fuerza, masa grasa y dolor \\ Combined resistance and aerobic training in Elderly: effects on functional performance, strength, fat mass and pain perception
}

*Nicolás Fernández-Martínez, **Carlos Pozo-Bohórquez, *Vanesa Sánchez-Canales *CEU Cardenal Spínola (España), **Almazara Club (España)

Resumen. Introducción: La sociedad está envejeciendo, y se estima que este segmento seguirá aumentando en los próximos años. Objetivo: Examinar los efectos que producen 5 semanas de entrenamiento en el rendimiento funcional, fuerza de agarre, perímetro de cintura, masa grasa y percepción de dolor general. Método: 28 sujetos (edad e» 55 años), sedentarios, participaron en un programa de entrenamiento de fuerza y entrenamiento aeróbico, de 5 semanas, 5 sesiones semanales de 50-60 minutos de duración. Resultados: Existen diferencias estadísticamente significativas entre los valores previos y posteriores al programa. La magnitud de estas diferencias puede considerarse grande en el Timed Up and Go Test $(-27.3 \%, p=0.000, d=3.01)$, 30Second Chair Stand Test $(+42 \%, \mathrm{p}=0.000, \mathrm{~d}=1.77)$ y percepción de dolor día $(-54.7 \%, \mathrm{p}=0.000, \mathrm{~d}=1.27)$ y noche $(-$ $58.2 \%, \mathrm{p}=0.000, \mathrm{~d}=1.02)$. Conclusión: Tras la aplicación del programa de entrenamiento concurrente descrito, todas las variables analizadas han evolucionado favorablemente. Dichas variables están directamente relacionadas con la composición corporal, calidad de vida, reducción del dolor, así como mejora de fuerza para las actividades cotidianas de las personas mayores.

Palabras clave: Envejecimiento, Composición corporal, Entrenamiento concurrente, Deterioro funcional, Calidad de vida.

\begin{abstract}
Introduction: Society is aging, and it is estimated that this segment will continue to grow in the coming years. Objectives: To examine the effects of 5 weeks of training on functional performance, grip strength, waist circumference, fat mass and general pain perception.Methods: 28 subjects (age e» 55 years), sedentary, participated in a 5-week strength and aerobic training program, 5 weekly sessions of 50-60 minutes duration. Results: There are statistically significant differences between pre and post program values. The magnitude of these differences can be considered large in theTimed Up and Go Test $(-27.3 \%, p=0.000, d=3.01), 30-$ Second Chair Stand Test $(+42 \%, \mathrm{p}=0.000, \mathrm{~d}=1.77)$ and pain perception day $(-54.7 \%$, $\mathrm{p}=0.000, \mathrm{~d}=1.27)$ and night $(-58.2 \%, \mathrm{p}=0.000, \mathrm{~d}=1.02)$. Conclusion: After the application of described concurrent training program, all analyzed variables have evolved favorably. Such variables are directly related to body composition, quality of life, pain reduction, as well as to improvement of strenght for the elderly's daily activities.
\end{abstract}

Keywords: Aging, Body Composition, Concurrent training, Functional impairment, Quality of life.

\section{Introducción}

La sociedad está envejeciendo progresivamente, y se estima que este segmento de la población seguirá aumentando en los próximos años (Sander et al., 2015).

Sin embargo, un aumento en la duración de la vida no significa necesariamente una mayor duración de la salud, siendo muestra de ello el aumento en las últimas décadas de las llamadas afecciones relacionadas con la edad (Valenzuela et al., 2019). De hecho, el envejecimiento se acompaña de una pérdida progresiva de masa muscular y función física deficiente denominada sarcopenia, que afecta drásticamente el estado de salud

Fecha recepción: 30-12-20. Fecha de aceptación: 22-08-21

Nicolás Fernández Martínez

nfernandez@ceuandalucia.es y la calidad de vida de las personas (Cruz-Jentoft et al., 2019).

En base a ello, el mantenimiento de la potencia muscular es un factor clave en el desempeño de las tareas cotidianas como subir escaleras, levantarse de una silla y caminar, así como para disminuir la probabilidad de caídas en las poblaciones de más edad (Alcañiz \& González-Moro, 2020; Arnold et al., 2010).

La investigación actual ha demostrado que el entrenamiento de fuerza es una intervención poderosa para combatir la pérdida de fuerza y masa muscular (sarcopenia), la vulnerabilidad fisiológica y sus consecuencias sobre el funcionamiento físico, la movilidad, la independencia y manejo de las enfermedades crónicas (Fragala et al., 2019; Miranda-Aguilar et al., 2020). Cabe destacar que Pinto et al. (2014) lograron mejorar la fuerza y pruebas de rendimiento funcional en adultos mayores sedentarios con sólo 6 semanas de entrena- 
miento de fuerza, 2 sesiones/semana.

Además, el entrenamiento de fuerza ayuda a conservar la masa magra mientras se reduce la grasa corporal (Miller et al., 2018; Mora et al., 2020; Sardeli et al., 2018), por lo que se optimiza la composición corporal y la salud metabólica.

Respecto al entrenamiento aeróbico, se ha demostrado que contrarresta efectos negativos del envejecimiento; mejora la disfunción mitocondrial (Cartee et al., 2016), promueve la capilarización del músculo esquelético (Gavin et al., 2007), mejora la función endotelial (Thomas et al., 2012), así como la función cardiorrespiratoria (como suele reflejarse en los aumentos en VO2 Max) (Chodzko-Zajko et al., 2009).

En consonancia con lo anterior, se considera que el entrenamiento concurrente es la mejor modalidad para atenuar el declive funcional, neuromuscular y cardiometabólico relacionado con la edad (Eduardo L. Cadore et al., 2011; Ferrari et al., 2016; Izquierdo et al., 2004; Moghadam et al., 2020; Wilhelm et al., 2014).

Los estudios previos con entrenamiento concurrente en adultos mayores son de al menos 8 semanas y con una frecuencia de 2-3 sesiones/semana (Agner et al., 2018; Eduardo Lusa Cadore et al., 2018; Morente-Oria et al., 2020; Neves et al., 2018; Shiotsu \&Yanagita, 2018; Timmons et al., 2018), por lo que nuestro propósito fue comprobar los efectos de 5 semanas a una frecuencia mayor (5 sesiones/semana).

Por tanto, el objetivo del estudio fue examinar los efectos que producen 5 semanas de entrenamiento en el rendimiento funcional, fuerza de agarre, perímetro de cintura, masa grasa y percepción de dolor general.

\section{Material y método}

\section{Participantes}

El tipo de muestreo fue no probabilístico por conveniencia. Se reclutaron 93 sujetos mayores, hombres y mujeres, todos sedentarios (que no habían realizado entrenamiento aeróbico o de fuerza de forma regular y sistemática durante al menos 1 año antes del estudio). La inscripción fue voluntaria y se obtuvo el consentimiento informado por escrito de cada participante. Se adoptaron los siguientes criterios de inclusión: hombres y mujeres mayores de 55 años y sedentarios.

Los criterios de exclusión fueron los siguientes: discapacidad física, cáncer, ictus, infartos y diabetes con uso de insulina. Los procedimientos cumplieron con la Declaración de Helsinki y el protocolo experimental fue aprobado por el Comité de Ética de la Universidad
Católica de Brasilia (protocolo no 235/2010). Después de la evaluación de elegibilidad, 28 sujetos mayores fueron seleccionados para participar (edad: 63,97 $\pm 6,58$; peso: 74,45 $\pm 11,23 \mathrm{~kg}$; altura: $160,1 \pm 8,50 \mathrm{~cm}$ ).

\section{Procedimientos}

Participaron en un programa de entrenamiento de fuerza y entrenamiento aeróbico, donde el de fuerza tuvo el mayor protagonismo. Se realizó durante 5 semanas, con una frecuencia de 5 sesiones/semana (de lunes a viernes). La duración de las sesiones fue de 5060 minutos, diseñadas y supervisadas en todo momento por un especialista Graduado en Ciencias de la Actividad Física y del Deporte. Se obtuvo un consentimiento informado por escrito de cada participante.

Dicho programa se llevó a cabo en las instalaciones de Almazara Club de Utrera (Sevilla). Se realizó una revisión estándar de la historia clínica de cada paciente para evaluar las patologías/comorbilidades prevalentes. Con estos datos, se formaron grupos de 3 personas de características similares. Se realizaron una serie de pruebas de valoración en la primera sesión (pre-test) y en la última (post-test).

Se realizaron 3 días de entrenamiento de fuerza (tabla 1) en el que predominaban ejercicios multiarticulares, con el objetivo de la mayor transferencia posible hacia las actividades cotidianas. Para diseñar el entrenamiento nos basamos en el posicionamiento de la National Strength and Conditioning Association sobre en entrenamiento de fuerza en personas mayores (Fragala et al., 2019). Los ejercicios para miembros superiores consistieron en tracciones (remos unilaterales y bilaterales) y empujes (press de pectoral horizontal en máquina), predominando los de tracción. Para miembros inferiores se realizaron sentadillas (adaptadas), leg extension y elevaciones de talón (patrón de flexión plantar).

También se focalizó en el entrenamiento del CORE, donde se utilizaron los patrones de extensión de cadera y ejercicios isométricos (planchas adaptadas).

La intensidad del entrenamiento de fuerza se prescribió mediante la escala de Borg modificada (0-10), pidiéndoles que llegaran a 6-7 (Morishita et al., 2019).

Se realizaron 3 series de 10 repeticiones por ejercicio, evitando llegar al fallo muscular (Eduardo Lusa Cadore et al., 2018; Neves et al., 2018). Se insistió a los participantes a evitar la maniobra de Valsalva durante la ejecución de los ejercicios. Las cargas de los ejercicios (entendida como el peso, ya que las series y repeticiones eran fijas) prácticamente permaneció estable des- 
pués del tercer día (una vez aprendida la técnica correcta), excepto algunos casos en los que se subió, para poder llegar a un RPE de 6-7.

\begin{tabular}{|c|c|c|}
\hline Ejercicios de fuerza & Series $\mathrm{x}$ repeticiones & Descanso \\
\hline Remo bilateral en polea (agarre neutro) & $3 \times 10$ & 1 ' \\
\hline Press de pectoral horizontal en máquina & $3 \times 10$ & $1^{\prime}$ \\
\hline Remo unilateral en polea & $3 \times 10$ & $1^{\prime}$ \\
\hline Sentadillas (adaptadas) & $3 \times 10$ & 1 ' \\
\hline Leg extension en máquina & $3 \times 10$ & 1 ' \\
\hline Elevaciones de talón & $3 \times 10$ & 1 ' \\
\hline Puente de glúteos & $3 \times 10$ & 1 ' \\
\hline Pull through en polea & $3 \times 10$ & 1 ' \\
\hline Planchas (adaptadas) & $10 \times 15 "$ & $20 "$ \\
\hline
\end{tabular}

En los 2 días restantes, se combinaban períodos de caminata en cinta de 15-20 minutos con ejercicios de fuerza con menor carga (ambas modalidades con una intensidad de 4-5 en Escala de Borg) (Noble et al., 1983). Las semanas 1 y 2, los sujetos caminaron 15 minutos en la cinta, y las 3 semanas restantes, caminaron 20 minutos.

\section{Instrumento}

Para evaluar el rendimiento funcional se utilizaron las pruebas Timed Up and Go Test (Savva et al., 2013), 30-Second Chair StandTest (Rikli \& Jones, 2013) y Back Scratch Test (Rikli \& Jones, 2013).

También se evaluaron otros parámetros como Fuerza de agarre (medida con dinamómetro de prensión manual) (García-Hermoso et al., 2018; Jochem et al., 2019; Kim et al., 2019), Perímetro de cintura (Ashwell et al., 2012; Schneider et al., 2010), Kilogramos de grasa (estimado por la mañana en ayunas con impedancia bioeléctrica Tanita BC-1000) (Sergi et al., 2017), Percepción de dolor general durante el día y durante la noche (evaluado con Escala Verbal Numérica) (Kremer et al., 1981).

\section{Análisis estadístico}

Los datos se procesaron con el paquete estadístico SPSS 24.0.

Para responder al problema planteado previamente se ha realizado en primer lugar un estudio descriptivo de las variables, seguido de la aplicación de contrastes paramétricos y no paramétricos, dependiendo de la normalidad o no (y homocedasticidad o no) de las variables analizadas.

En el estudio descriptivo se observa que la evolución de la media de todas las variables es favorable tras la realización del programa de entrenamiento. Se calcula, además, el tamaño del efecto para cada variable mediante el cálculo de la d de Cohen (Lakens, 2013) para muestras relacionadas sin grupo de control (tabla 2):

$$
d=\frac{\bar{X}_{\text {post }}-\bar{X}_{\text {pre }}}{S_{\text {post }}}
$$

Categorizando los valores del mismo en efecto pequeño (0.2), medio (0.5) y grande (0.8).

Tabla 2 .

Valores de las variables (media \pm desviación típica) pre y post, tamaño del efecto.

\begin{tabular}{|c|c|c|c|}
\hline & $P R E$ & POST & $d$ \\
\hline Agilidad & $7.38 \pm 1.24$ & $5.36 \pm .67$ & $3.01 * * *$ \\
\hline Fuerza y potencia de piernas & $13.54 \pm 3.47$ & $19.21 \pm 3.21$ & $1.77 * * *$ \\
\hline Fuerza de agarre izquierda & $26.56 \pm 8.88$ & $27.31 \pm 8.08$ & $.09 *$ \\
\hline Fuerza de agarre derecha & $27.87 \pm 10.07$ & $29.70 \pm 9.30$ & $.20 *$ \\
\hline Percepción de dolor (día) & $5.29 \pm 2.73$ & $2.39 \pm 2.28$ & $1.27 * * *$ \\
\hline Percepción de dolor (noche) & $4.36 \pm 3.57$ & $1.82 \pm 2.50$ & $1.02 * * *$ \\
\hline Perímetro cintura & $98.96 \pm 11.08$ & $95.07 \pm 10.62$ & $.37 *$ \\
\hline Kg de grasa & $28.06 \pm 7.92$ & $26.97 \pm 7.64$ & $.14 *$ \\
\hline Flexibilidad superior izquierda & $-12.39 \pm 10.00$ & $-8.07 \pm 9.08$ & $.48 * *$ \\
\hline Flexibilidad superior derecha & $-6.75 \pm 9.00$ & $-3.21 \pm 8.73$ & $.41 * *$ \\
\hline
\end{tabular}

$\mathrm{Al}$ contar con una muestra reducida de sujetos masculinos no se considera apropiado establecer un estudio comparativo por género.

Tras el estudio descriptivo se realiza la prueba de normalidad de Kolmogorov-Smirnov de la que se deduce que no puede asumirse la normalidad de las variables fuerza de agarre derecha $\operatorname{PRE}(\mathrm{F}=0,172 ; \mathrm{p}=0.033)$ y POST ( $\mathrm{F}=0.206 ; \mathrm{p}=0.004)$, percepción de dolor día $\operatorname{PRE}(\mathrm{F}=0.199, \mathrm{p}=0.006)$ y $\operatorname{POST}(\mathrm{F}=0.193, \mathrm{p}=0.009)$, percepción de dolor noche PRE $(\mathrm{F}=0.210, \mathrm{p}=0.003) \mathrm{y}$ $\operatorname{POST}(\mathrm{F}=0.236, \mathrm{p}=0.000)$ y perímetro de cintura $\mathrm{PRE}$ $(\mathrm{F}=0.177, \mathrm{p}=0.025)$.

También se realiza la prueba de Levene para la igualdad de varianzas en las variables que han presentado normalidad en la prueba K-S, obteniéndose homogeneidad de varianzas en todas ellas salvo en la variable Timed Up and GoTest (W=11.277, $\mathrm{p}=0.001)$. Por tanto, se incluye esta variable en los estudios no paramétricos.

Finalmente, se aplican pruebas no paramétricas (Test de Wilcoxon) para el análisis de las variables Timed Up and Go Test, fuerza de agarre derecha, dolor (día y noche) y perímetro de cintura. Para el resto de variables se aplican contrastes paramétricos (prueba T para muestras relacionadas).

\section{Resultados}

Se ha realizado la Prueba T para muestras relacionadas en aquellas variables que cumplen los supuestos de normalidad y homocedasticidad, obteniendo diferencias significativas con tamaño del efecto grande en 30-Second Chair Stand Test $(\mathrm{t}=-11.901, \mathrm{p}=0.000, \mathrm{~d}=1.77)$, con tamaño del efecto medio en el Back Scratch Test del lado izquierdo $(\mathrm{t}=-8.083, \mathrm{p}=0.000, \mathrm{~d}=0.48)$ y derecha 
( $t=-7.035, p=0.000, d=0.41)$, y con tamaño del efecto pequeño en $\mathrm{kg}$ de grasa $(\mathrm{t}=5.538, \mathrm{p}=0.000, \mathrm{~d}=0.14)$ (Tablas 2 y 3).

\begin{tabular}{|c|c|c|c|}
\hline & Media \pm DT & $\mathrm{T}$ & Sig. (bilateral) \\
\hline Fuerza y potencia & $-5.68 \pm 2.52$ & -11.90 & $.000 *$ \\
\hline Fuerza de agarre izquierda & $-.75 \pm 3.17$ & -1.25 & .223 \\
\hline Kg de grasa & $1.09 \pm 1.04$ & 5.54 & $.000 *$ \\
\hline Flexibilidad superior izquierda & $-4.32 \pm 2.83$ & -8.08 & $.000 *$ \\
\hline Flexibilidad superior derecha & $-3.54 \pm 2.66$ & -7.04 & $.000 *$ \\
\hline
\end{tabular}

Para el resto de variables se ha realizado la Prueba de rangos de Wilcoxon obteniéndose diferencias estadísticamente significativas entre los valores previos y posteriores al entrenamiento en las siguientes variables: con un tamaño del efecto grande en el Timed Up and GoTest $(Z=-4.623, p=0.000, d=3.01)$, percepción de dolor día $(Z=-4.307, \mathrm{p}=0.000, \mathrm{~d}=1.27)$ y noche $(\mathrm{Z}=$ $3.838, \mathrm{p}=0.000, \mathrm{~d}=1.02)$, y con un tamaño del efecto pequeño en perímetro de cintura $(Z=-4.630, p=0.000$, $\mathrm{d}=0.37)$ y en fuerza de agarre derecha $(\mathrm{Z}=-3.018$, $\mathrm{p}=0.003, \mathrm{~d}=0.2)($ Tablas $1 \mathrm{y} 4)$.

\begin{tabular}{|c|c|c|}
\hline & $\mathrm{Z}$ & Sig. Asintót. (bilateral) \\
\hline Agilidad & $-4.62^{b}$ & $.000 *$ \\
\hline Fuerza de agarre derecha & $-3.02^{\mathrm{c}}$ & $.003 *$ \\
\hline Percepción de dolor (día) & $-4.31^{\mathrm{b}}$ & $.000 *$ \\
\hline Percepción de dolor (noche) & $-3.84^{\mathrm{b}}$ & $.000 *$ \\
\hline Perímetro cintura & $-4.63^{\mathrm{b}}$ & $.000 *$ \\
\hline
\end{tabular}

de contraste.

\section{Discusión}

El propósito del estudio fue examinar los efectos que producen 5 semanas de entrenamiento en el rendimiento funcional, fuerza de agarre, perímetro de cintura, masa grasa y percepción de dolor general.

Los resultados de este estudio respaldan la hipótesis de que 5 semanas de entrenamiento (en personas mayores previamente sedentarias) mejoran la media de los test realizados.

En cuanto al Timed Up and Go Test, se obtuvieron mejoras considerables ( $\mathrm{d}=3.01)$, en consonancia con los resultados de Pinto et al. (2014) que observaron beneficios en dicho test tras la aplicación de un programa de entrenamiento de fuerza de miembros inferiores en adultos mayores de tan sólo 6 semanas (duración similar a nuestra intervención), 2 sesiones/semana.

Centrándonos en el 30-Second Chair Stand Test, se observaron grandes cambios positivos ( $d=1.77)$. En este sentido, en el mismo estudio de Pinto et al. (2014) también encontraron mejoras en el rendimiento de esta prueba. Resultados similares obtuvieron Barbalho et al.
(2017) tras aplicar un programa de entrenamiento de fuerza de cuerpo completo a mujeres mayores durante 12 semanas.

Por otro lado, cabe destacar que en nuestra intervención no se usaron ejercicios de flexibilidad, pero aun así el Back Scratch Test también se obtuvieron mejoras $(\mathrm{d}=0.48$ en parte izquierda $\mathrm{y} d=0.41$ en parte derecha). Dichos resultados concuerdan con el estudio de Carvalho, Marques, \& Mota (2009) en el que se combinó entrenamiento de fuerza, aeróbico y de flexibilidad. Este cambio favorece la realización de actividades como peinarse, ducharse, etc.

También se encontraron pequeños aumentos en la fuerza de prensión manual de la mano izquierda $(\mathrm{d}=0.09)$ y derecha $(\mathrm{d}=0.20)$. Este parámetro está estrechamente relacionado con el riesgo de muerte tanto en personas aparentemente sanas (García-Hermoso et al., 2018) como en personas con enfermedades (Jochem et al., 2019).

Los test anteriores reflejan movimientos cotidianos que pueden verse limitados en el adulto mayor, y, por tanto, el aumento del rendimiento en dichas pruebas contribuye a una mejor calidad de vida en esta población.

Centrándonos en la composición corporal cabe subrayar la disminución de la masa grasa $(\mathrm{d}=0.14)$. Debido a que la impedancia bioeléctrica tiene cierto error de estimación por varios factores (Sergi et al., 2017), no se puede asegurar que se haya producido tal disminución de forma exacta. Por otro lado, se podría afirmar que se ha mantenido la masa magra durante la pérdida de grasa gracias al entrenamiento (Miller et al., 2018; Sardeli et al., 2018), así como una mejora de la calidad muscular (Cunha et al., 2018).

En cuanto a las medidas antropométricas, la reducción del perímetro de la cintura $(\mathrm{d}=0.37)$ ha disminuido el riesgo de enfermedades cardiovasculares (Ramírez-Vélez et al., 2019), ya que la grasa a nivel abdominal es la más peligrosa porque compromete a los órganos vitales (Ashwell et al., 2012; Schneider et al., 2010).

La percepción del dolor general durante el día $(\mathrm{d}=1.27)$ y la noche $(\mathrm{d}=1.02)$ disminuyó sustancialmente. Estos resultados están en consonancia con diversos estudios, donde disminuyen o eliminan el dolor lumbar (Ciolac \& Rodrigues-da-Silva, 2016; Cortell-Tormo et al., 2018) después de un programa de entrenamiento de fuerza. En la revisión de Tanaka, Ozawa, Kito, \& Moriyama (2013) se concluyó que tanto el entrenamiento de fuerza como el aeróbico eran efectivos para redu- 
cir el dolor de rodilla en personas con osteoartritis. Este hecho es de especial relevancia en nuestro estudio, puesto que la mayoría de los participantes presentaban esta dolencia.

Además, el meta-análisis de Nascimento et al., 2018 concluyó que el fortalecimiento de la musculatura de la rodilla y cadera era más efectivo para reducir el dolor de rodilla que fortalecer sólo la musculatura de la rodilla. Cabe destacar que nuestro programa incluyó el fortalecimiento de la musculatura de ambas articulaciones.

Curiosamente, muchas personas informaron de una disminución de dolores e inflamación en zonas que no habían sido entrenadas de forma específica. En los últimos años está emergiendo gran cantidad de literatura cientifica en la que se explica el rol de las mioquinas, las citoquinas que produce el músculo que poseen un potente efecto antiinflamatorio. Estas moléculas se segregan gracias a la contracción muscular inducida por el ejercicio. Lo interesante de las mioquinas es que no sólo actúan a nivel local, sino que se trasladan por la sangre hacia otros órganos y tejidos (Ellingsgaard et al., 2019; Huh, 2018; Ribeiro et al., 2015). Esto podría explicar en parte este fenómeno.

El estudio realizado tiene algunas limitaciones. Como primera limitación, no se hicieron analíticas de sangre para observar posibles cambios en parámetros relacionados con la salud cardio-metabólica. Como segunda limitación, en este estudio hubo participantes de sexo masculino y femenino, pudiendo interferir en los resultados, puesto que los efectos del entrenamiento pueden diferir entre géneros.

Como futura línea de investigación, se podría replicar este mismo estudio con una muestra mayor y más prolongado en el tiempo.

\section{Conclusiones}

Tras la aplicación del programa de entrenamiento concurrente descrito con una duración de sólo 5 semanas, todas las variables analizadas han evolucionado favorablemente. Dichas variables están directamente relacionadas con la composición corporal, calidad de vida, reducción del dolor, así como mejora de fuerza para las actividades cotidianas de las personas mayores.

\section{Referencias}

Agner,V.F.C., Garcia, M.C.,Taffarel,A.A., Mourão, C.B.,daSilva, I.P., da Silva, S. P., Peccin, M. S., \& Lombardi, I. (2018). Effects of concurrent training on muscle strength in older adults with metabolic syndrome:A randomized controlled clinical trial. Archives of Gerontology and Geriatrics, 75(December 2017), 158-164.https://doi.org/10.1016/j.archger.2017.12.011

Alcañiz, R. N., \& González-Moro, I. M. (2020).Valoración del grado de deterioro funcional y fragilidad en adultos mayores activos. Retos, 38, 576-581.

Arnold, C.M.,Warkentin, K.D., Chilibeck,P.D., \& Magnus, C.R. A.(2010).Thereliability and validity of handheld dynamometry for the measurement of lower-extremity muscle strength in older adults. Journal of Strength and Conditioning Research, 24(3), 815-824. https://doi.org/10.1519/ JSC.0b013e3181aa36b8

Ashwell, M., Gunn, P., \& Gibson, S. (2012).Waist-to-height ratio is a better screening tool than waist circumference and BMI for adult cardiometabolic risk factors: systematic review and meta-analysis. Obesity Reviews, 13(3), 275-286. https:// doi.org/10.1111/j.1467-789X.2011.00952.x

Barbalho,M.deS.M., Gentil,P., Izquierdo, M.,Fisher,J.,Steele,J., \& Raiol, R. deA.(2017). There are no no-responders to low or high resistance training volumes among older women. Experimental Gerontology, 99, 18-26.https://doi.org/10.1016/ j.exger.2017.09.003

Cadore, Eduardo L., Pinto, R. S., Pinto, S. S., Alberton, C. L., Correa, C. S., Tartaruga, M. P., Silva, E. M.,Almeida, A. P.V., Trindade, G.T., \& Kruel,L. F. M. (2011). Effects of strength, endurance, and concurrent training on aerobic power and dynamic neuromuscular economy in elderly men. Journal of Strength and Conditioning Research, 25(3), 758-766. https:/ / doi.org/10.1519/JSC.0b013e318207ed66

Cadore,Eduardo Lusa, Menger,E.,Teodoro,J.L.,daSilva,L.X.N., Boeno,F.P.,Umpierre,D., Botton, C.E.,Ferrari,R., Cunha, G. dos S., Izquierdo, M., \& Pinto, R. S. (2018). Functional and physiological adaptationsfollowing concurrent training using sets with and without concentric failure in elderly men: A randomized clinical trial. Experimental Gerontology, 110, 182190.https://doi.org/10.1016/j.exger.2018.06.011

Cartee, G. D., Hepple, R. T., Bamman, M. M., \& Zierath, J. R. (2016). Exercise Promotes Healthy Aging of Skeletal Muscle. In Cell Metabolism (Vol. 23, Issue 6, pp. 1034-1047). Cell Press. https://doi.org/10.1016/j.cmet.2016.05.007

Carvalho, M. J., Marques, E., \& Mota, J. (2009). Training and detraining effects on functional fitness after a multicomponent training in older women. Gerontology, 55(1), 41-48.https:// doi.org/10.1159/000140681

Chodłko-Zajko,W.J.,Proctor,D.N., FiataroneSingh,M.A.,Minson, C.T., Nigg, C. R., Salem, G. J., \& Skinner, J. S. (2009). Exercise and physical activity for older adults. In Medicine and Science in Sports and Exercise (Vol.41, Issue 7, pp. 1510-1530).https:// doi.org/10.1249/MSS.0b013e3181a0c95c

Ciolac,E. G., \& Rodrigues-da-Silva,J.M. (2016).ResistanceTraining asaTool for Preventing andTreating Musculoskeletal Disorders. 
In Sports Medicine (Vol. 46, Issue 9, pp. 1239-1248). https: / / doi.org/10.1007/s40279-016-0507-z

Cortell-Tormo, J. M., Sánchez, P.T., Chulvi-Medrano, I., TortosaMartínez, J., Manchado-López, C., Llana-Belloch,S., \& PérezSoriano, P. (2018). Effects of functional resistance training on fitness and quality of life in females with chronic nonspecific low-back pain. Journal of Back and Musculoskeletal Rehabilitation, 31(1), 95-105. https://doi.org/10.3233/BMR-169684

Cruz-Jentoft, A. J., Bahat, G., Bauer, J., Boirie,Y., Bruyère, O., Cederholm,T., Cooper, C., Landi, F., Rolland,Y., Sayer,A.A., Schneider,S.M., Sieber, C. C., Topinkova, E.,Vandewoude, M., Visser, M.,Zamboni, M., Bautmans, I., Baeyens, J.P., Cesari, M., ... Schols, J. (2019). Sarcopenia: Revised European consensus on definition and diagnosis. Age and Ageing, 48(1), 16-31.https://doi.org/10.1093/ageing/afy169

Cunha, P. M., Nunes, J. P., Tomeleri, C. M., Nascimento, M.A., Schoenfeld, B. J., Antunes, M., Gobbo, L.A., Teixeira, D., \& Cyrino,E. S. (2018). ResistanceTraining PerformedWith Single and Multiple Sets Induces Similar Improvements in Muscular Strength, Muscle Mass, Muscle Quality, and IGF-1 in OlderWomen. Journal of Strength and Conditioning Research, 1. https: / /doi.org/10.1519/jsc.0000000000002847

Ellingsgaard, H., Hojman, P., \& Pedersen, B. K. (2019). Exercise and health - emerging roles of IL-6. In Current Opinion in Physiology (Vol. 10, pp. 49-54). Elsevier. https://doi.org/ 10.1016/j.cophys.2019.03.009

Ferrari, R., Fuchs, S.C., Kruel, L. F.M., Cadore,E. L., Alberton, C. L., Pinto, R. S., Radaelli, R., Schoenell, M., Izquierdo, M., Tanaka, H., \& Umpierre, D. (2016). Effects of different concurrent resistance and aerobic training frequencies on muscle power and muscle quality in trained elderly men: A randomized clinical trial. Aging and Disease, 7(6), 697-704. https://doi.org/10.14336/AD.2016.0504

Fragala,M.S., Cadore, E. L., Dorgo, S., Izquierdo, M., Kraemer,W. J.,Peterson, M.D., \& Ryan, E.D. (2019). ResistanceTraining for Older Adults: Position Statement From the National Strength and ConditioningAssociation. Journal of Strength and Conditioning Research, 33(8), 2019-2052. https://doi.org/ 10.1519/JSC.0000000000003230

García-Hermoso,A., Cavero-Redondo,I., Ramírez-Vélez, R., Ruiz, J.R., Ortega,F.B., Lee, D. C., \& Martínez-Vizcaíno,V.(2018). Muscular Strengthas a Predictor of All-Cause Mortality in an Apparently Healthy Population:A SystematicReview and MetaAnalysis of Data From Approximately 2 Million Men and Women. Archives of Physical Medicine and Rehabilitation, 99(10), 2100-2113.e5. https://doi.org/10.1016/ j.apmr.2018.01.008

Gavin,T.P., Ruster, R.S., Carrithers,J.A.,Zwetsloot,K.A., Kraus, R. M., Evans, C.A., Knapp, D. J., Drew, J. L., McCartney, J. S., Garry, J. P., \& Hickner, R. C. (2007). No difference in the skeletalmuscleangiogenicresponse toaerobic exercise training between young and aged men. Journal of Physiology, 585(1),
231-239. https:/ / doi.org/10.1113/jphysiol.2007.143198 Huh,J.Y.(2018).The roleof exercise-induced myokinesin regulating metabolism. In Archives of Pharmacal Research (Vol.41, Issue 1, pp. 14 -29). https://doi.org/10.1007/s12272-017-0994$\mathrm{y}$

Izquierdo, M., Ibañez, J., Häkkinen, K., Kraemer,W. J., Larrión, J. L., \& Gorostiaga, E. M. (2004). Once Weekly Combined Resistance and CardiovascularTraining in Healthy Older Men. Medicine and Science in Sports and Exercise, 36(3), 435-443. https://doi.org/10.1249/01.MSS.0000117897.55226.9A Jochem, C., Leitzmann, M., Volaklis, K.,Aune, D., \& Strasser, B. (2019).Association Between Muscular Strengthand Mortality in Clinical Populations: A SystematicReview and Meta-Analysis. Journal of the American Medical DirectorsAssociation, 20(10), 1213 1223. https://doi.org/10.1016/j.jamda.2019.05.015

Kim, G. R.,Sun,J.,Han, M.,Park,S., \& Nam, C.M. (2019). Impact of handgrip strength on cardiovascular, cancer and all-cause mortalityin the Korean longitudinal study of ageing. BMJ Open, 9(5). https://doi.org/10.1136/bmjopen-2018-027019

Kremer,E.,Atkinson, J.H., \& Ignelzi, R. J. (1981). Measurement of pain: patient preference does not confound pain measurement. Pain, 10(2), 241-248. http:// www.ncbi.nlm.nih.gov/pubmed/7267140

Lakens,D. (2013). Calculating and reporting effect sizes to facilitate cumulative science:A practical primerfor t-tests and ANOVAs. Frontiers in Psychology, 4(NOV). https: / / doi.org/10.3389/ fpsyg.2013.00863

Miller, T., Mull, S., Aragon, A.A., Krieger, J., \& Schoenfeld, B. J. (2018). Resistance training combined with diet decreases body fat while preserving leanmassindependent of restingmetabolic rate:A randomized trial. International Journal of Sport Nutrition and Exercise Metabolism, 28(1), 46-54. https://doi.org/ 10.1123/ijsnem.2017-0221

Miranda-Aguilar, D., Valdés-Badilla, P., Herrera-Valenzuela, T., Guzmán-Muñoz, E., Magnani Branco, B. H., MéndezRebolledo, G., \& López-Fuenzalida,A. (2020). Elasticbands or gym equipment for the training of older adults? Retos, 37 , 370-378.

Moghadam, B. H., Bagheri, R., Ashtary-Larky, D., Tinsley, G. M., Eskandari, M.,Wong,A., Moghadam, B. H., Kreider, R. B., \& Baker, J.S. (2020). The Effects of ConcurrentTraining Order on Satellite Cell-Related Markers, Body Composition, Muscular and Cardiorespiratory Fitness in Older Men with Sarcopenia. Journal of Nutrition, Health and Aging, 24(7), 796 804. https: / /doi.org/10.1007/s12603-020-1431-3

Mora, R. M. S., Oliver,A.J. S., Carmona,W. S., \& Jurado, J.A. G. (2020). Effect of a physical exercise program on physical fitness and visceral fat in people with obesity. Retos, 2041(39), 723730. https: / / doi.org/10.47197/retos.v0i39.78997

Morente-Oria, H., Ruiz-Montero, P. J., Chiva-Bartoll, Ó., \& González-Fernández, F. T. (2020). Effects of 8-weeks concurrent strengthandAerobic trainingon body composition, 
physiological and cognitive performancein older adultwomen. Sustainability (Switzerland), 12(5), 1-14. https://doi.org/ $10.3390 /$ su12051944

Morishita,S.,Tsubaki,A., Nakamura, M., Nashimoto,S., Fu, J.B., \& Onishi, H. (2019). Rating of perceived exertion on resistance training in elderly subjects. Expert Review of CardiovascularTherapy, 17(2), 135-142. https://doi.org/10.1080/ 14779072.2019.1561278

Nascimento, L. R., Teixeira-Salmela,L.F., Souza, R. B., \& Resende, R. A. (2018). Hip and knee strengthening is more effective than kneestrengthening alonefor reducing pain and improving activity in individuals with patellofemoral pain: A systematic review with meta-analysis. Journal of Orthopaedic and Sports Physical Therapy, 48(1), 19-31. https: / /doi.org/10.2519/ jospt.2018.7365

Neves, L.X.daS.,Teodoro,J.L., Menger,E.,Lopez,P., Grazioli,R., Farinha,J., Moraes, K., Bottaro, M.,Pinto, R. S., Izquierdo, M., $\&$ Cadore, E. L. (2018). Repetitions to failure versus not to failure during concurrent training in healthy elderly men:A randomized clinical trial. Experimental Gerontology, 108, 18-27. https://doi.org/10.1016/j.exger.2018.03.017

Noble, B. J., Borg, G.A.V., Jacobs, I., Ceci, R., \& Kaiser,P.(1983).A category-ratio perceived exertion scale: Relationship toblood and muscle lactates and heart rate. Medicine and Science in Sports and Exercise, 15(6), 523-528. https://doi.org/10.1249/ 00005768-198315060-00015

Pinto, R. S., Correa, C. S., Radaelli, R., Cadore, E. L., Brown, L. E., \& Bottaro, M. (2014). Short-term strength training improves muscle quality and functional capacity of elderly women. Age, 36(1), 365-372. https://doi.org/10.1007/s11357-0139567-2

Ramírez-Vélez, R., Pérez-Sousa, M. Á., Izquierdo, M., CanoGutierrez, C.A., González-Jiménez, E., Schmidt-Riovalle, J., González-Ruíz, K., \& Correa-Rodríguez,M.(2019).Validation of surrogate anthropometric indices in older adults: What is the bestindicator of high cardiometabolicrisk factor clustering? Nutrients, 11(8). https://doi.org/10.3390/nu11081701

Ribeiro,A.S.,Tomeleri, C.M.,Souza,M.F.,Pina,F.L.C., Schoenfeld, B. J., Nascimento, M.A.,Venturini, D., Barbosa,D. S., \& Cyrino, E. S. (2015). Effect of resistance training on C-reactive protein, blood glucose and lipid profile in older women with differing levels of RT experience. $A G E, 37(6), 109$. https: / / doi.org/ 10.1007/s11357-015-9849-y

Rikli, R. E., \& Jones, C. J. (2013). Senior fitness testmanual. Choice Reviews Online, 39(06), 39-3447-39-3447. https:/ / doi.org/ 10.5860/ choice.39-3447

Sander, M., Oxlund, B., Jespersen,A., Krasnik,A., Mortensen,E. L., Westendorp, R. G. J., \& Rasmussen, L. J. (2015). The challenges of human population ageing. Age and Ageing, 44(2), 185-187. https: / /doi.org/10.1093/ageing/afu189

Sardeli,A.V.,Komatsu,T.R., Mori, M.A., Gáspari,A.F., \& ChaconMikahil, M.P.T.(2018). Resistance training prevents muscle loss induced by caloric restriction in obese elderly individuals: A systematic review and meta-analysis. In Nutrients (Vol. 10, Issue4). MDPIAG.https: //doi.org/10.3390/nu10040423 Savva, G. M., Donoghue, O.A., Horgan, F., O’Regan, C., Cronin, H., \& Kenny, R.A.(2013). Using timed up-and-gotoidentify frail members of the older population. Journals of Gerontology Series A Biological Sciences and Medical Sciences, 68(4), 441446. https://doi.org/10.1093/gerona/gls190

Schneider, H. J., Friedrich, N., Klotsche, J., Pieper, L., Nauck, M., John, U., Dörr, M., Felix,S., Lehnert, H., Pittrow,D., Silber,S., Völzke,H., Stalla, G. K., Wallaschofski,H., \&Wittchen,H. U. (2010).The predictive value of different measures of obesity for incident cardiovascular events and mortality. Journal of Clinical Endocrinology and Metabolism, 95(4), 1777-1785. https://doi.org/10.1210/jc.2009-1584

Sergi, G., De Rui, M., Stubbs, B., Veronese, N., \& Manzato, E. (2017). Measurement of lean body mass using bioelectrical impedance analysis: a consideration of the pros and cons. In Aging Clinical and Experimental Research (Vol. 29, Issue 4, pp. 591-597). https://doi.org/10.1007/s40520-016-0622-6 Shiotsu,Y., \&Yanagita, M. (2018). Comparisons of low-intensity versus moderate-intensity combined aerobic and resistance training on body composition, muscle strength, and functional performance in older women. Menopause, 25(6), 668-675. https: / /doi.org/10.1097/GME.0000000000001060

Tanaka, R., Ozawa, J., Kito, N., \& Moriyama,H.(2013).Efficacy of strengthening or aerobic exercise on pain relief in people with knee osteoarthritis: A systematic review and meta-analysis of randomized controlled trials. In Clinical Rehabilitation (Vol. 27, Issue 12, pp. 1059-1071). https://doi.org/10.1177/ 0269215513488898

Thomas,A.W., Davies, N.A., Moir, H.,Watkeys, L., Ruffino, J. S., Isa, S.A., Butcher,L. R., Hughes, M. G., Morris, K., \&Webb, R. (2012). Exercise-associated generation of PPARã ligands activates PPARã signaling events and upregulates genes related to lipid metabolism. Journal of Applied Physiology, 112(5), 806 815. https:/ /doi.org/10.1152/japplphysiol.00864.2011

Timmons, J.F., Minnock, D., Hone, M., Cogan, K. E., Murphy, J. C., \& Egan, B. (2018). Comparison of time-matched aerobic, resistance, or concurrent exercise training in older adults. In Scandinavian Journal of Medicine and Science in Sports (Vol. 28, Issue 11). https: / / doi.org/10.1111/sms.13254

Valenzuela, P.L., Castillo-García,A., Morales, J. S., Izquierdo, M., Serra-Rexach, J.A., Santos-Lozano, A., \& Lucia, A. (2019). Physical exercise in the oldest old. Comprehensive Physiology, 9(4), 1281-1304.https://doi.org/10.1002/cphy.c190002 Wilhelm,E.N., Rech,A., Minozzo, F., Botton, C.E., Radaelli, R., Teixeira, B. C., Reischak-Oliveira,A., \& Pinto, R. S. (2014). Concurrent strengthand endurance training exercise sequence doesnot affect neuromuscular adaptationsin older men. Experimental Gerontology, 60,207-214.https://doi.org/10.1016/ j.exger.2014.11.007 